\title{
Indirect comparison of NSAIDs for ankylosing spondylitis: Network meta-analysis of randomized, double-blinded, controlled trials
}

\author{
MEIDA FAN ${ }^{1,2 *}$, JIAN LIU $^{3 *}$, BINGCHENG ZHAO $^{3}$, XINYU WU $^{1}$, XUEFENG LI $^{4-6}$ and JIERUO GU ${ }^{1}$ \\ ${ }^{1}$ Department of Rheumatology, The Third Affiliated Hospital of Sun Yat-Sen University, Guangzhou, Guangdong 510630; \\ Departments of ${ }^{2}$ Rheumatology and ${ }^{3}$ Anesthesiology, Nanfang Hospital, Southern Medical University, Guangzhou, \\ Guangdong 510515; ${ }^{4}$ Department of Gastrointestinal Surgery, The Sixth Affiliated Hospital of Guangzhou Medical University, \\ Qingyuan People's Hospital, State Key Laboratory of Respiratory Disease, Sino-French Hoffmann Institute, School of \\ Basic Medical Sciences, Guangzhou Medical University, Guangzhou, Guangdong 511436; ${ }^{5}$ Shenzhen Following Precision \\ Medical Research Institute, Shenzhen Luohu People's Hospital, The Third Affiliated Hospital of Shenzhen University, \\ Shenzhen, Guangdong 518001; ${ }^{6}$ Key Laboratory of Regenerative Biology, Guangdong Provincial Key Laboratory of Stem \\ Cell and Regenerative Medicine, South China Institute for Stem Cell Biology and Regenerative Medicine, Guangzhou \\ Institutes of Biomedicine and Health, Chinese Academy of Sciences, Guangzhou, Guangdong 510530, P.R. China
}

Received April 3, 2019; Accepted November 15, 2019

DOI: $10.3892 / \mathrm{etm} .2020 .8564$

\begin{abstract}
Ankylosing spondylitis (AS) is a chronic inflammatory disease characterized by lower back pain, enthesitis and asymmetrical peripheral arthritis. Non-steroidal anti-inflammatory drugs (NSAIDs) are recommended as a first-line drug treatment for AS. The aim of the present study was to evaluate the efficacy and safety of NSAIDs in patients with active AS. A total of 9 randomized controlled trials focusing on 6 NSAIDs, including etoricoxib, celecoxib, meloxicam, diclofenac, naproxen and beta-D-mannuronic acid (M2000), were analyzed in the present study. The efficacy endpoints included total pain score, patients' global assessment of disease activity (PGA), Bath Ankylosing Spondylitis Functional Index (BASFI) and the rate of achieving an Assessment in
\end{abstract}

Correspondence to: Dr Jieruo Gu, Department of Rheumatology, The Third Affiliated Hospital of Sun Yat-Sen University, 600 Tianhe Road, Tianhe, Guangzhou, Guangdong 510630, P.R. China

E-mail: gujieruo@163.com

Dr Xuefeng Li, Department of Gastrointestinal Surgery, The Sixth Affiliated Hospital of Guangzhou Medical University, Qingyuan People's Hospital, State Key Laboratory of Respiratory Disease, Sino-French Hoffmann Institute, School of Basic Medical Sciences, Guangzhou Medical University, 195 Dongfeng Xi Road, Xinzao, Panyu, Guangzhou, Guangdong 511436, P.R. China

E-mail: xuefengli@gzhmu.edu.cn

*Contributed equally

Key words: ankylosing spondylitis, non-steroidal anti-inflammatory drugs, efficacy, safety, meta-analysis
Ankylosing Spondylitis 20\% response (ASAS20). The safety endpoints included total adverse events (AEs), gastrointestinal (GI) AEs, withdrawals due to AEs and serious AEs. NSAIDs were compared with the placebo and among themselves using Bayesian network meta-analysis, calculating mean differences (MDs) for continuous data and odds ratios for dichotomous data. The analysis revealed that all NSAIDs were significantly more effective in reducing pain severity than placebo (MDs between -17.49 and -25.99). Similarly, significant improvements in PGA, BASFI and ASAS20 were determined in patients receiving NSAIDs. Furthermore, etoricoxib was ranked as the most efficacious treatment for patients with AS. With regard to safety, there were no significant differences between NSAIDs and placebo in terms of total AEs, withdrawals due to AEs or serious AEs. Furthermore, no significant differences in AEs were identified between M2000 and the placebo. However, patients treated with diclofenac and naproxen had a higher risk of GI events than those taking placebo. In conclusion, the NSAIDs were all highly effective and well-tolerated in the treatment of AS. However, clinicians should take GI toxicity into account when prescribing NSAIDs.

\section{Introduction}

Ankylosing spondylitis (AS) is a chronic inflammatory disease characterized by lower back pain, enthesitis and asymmetrical peripheral arthritis (1). It predominantly affects the axial skeleton (2). Hip involvement usually causes severe functional impairment $(3,4)$. The disease commonly occurs in the second or third decade of life. Without adequate treatment, it may lead to disability and a significant decrease in quality of life $(5,6)$.

Non-steroidal anti-inflammatory drugs (NSAIDs) are commonly used to reduce pain and inflammation, and 
are recommended as first-line agents for the treatment of AS (7-9). Furthermore, continuous treatment with NSAIDs is preferred for symptomatic patients $(8,10,11)$. However, the use of NSAIDs may increase the risk of gastrointestinal (GI), cardiovascular and renal adverse events (AEs) (12-14). Traditional non-selective NSAIDs are associated with an increased risk of GI events due to their inhibition of cyclooxygenase (COX)-1 isoenzyme (15). Furthermore, COX-2 selective inhibitors may reduce GI toxicity but increase the risk of cardiovascular events $(16,17)$.

Several randomized controlled trials (RCTs) have indicated that in AS, COX-2 inhibitors, including etoricoxib and celecoxib, have comparable or superior efficacy and improved GI tolerability compared to non-selective NSAIDs (18-22). However, comprehensive comparisons among various NSAIDs are scarce. It is necessary to compare the benefits and disadvantages among the most commonly used NSAIDs. In the present study, an indirect comparison was performed using a Bayesian network meta-analysis to assess the efficacy and safety of NSAIDs in the treatment of AS.

\section{Materials and methods}

Literature search. A systematic literature search on PubMed, Embase, the Cochrane Library, China National Knowledge Infrastructure and WanFang databases (with entries up to August 12, 2019 considered) was performed without any restrictions regarding the region, publication date or language. Titles/abstracts were searched using logic combinations of the following terms: ('ankylosing spondylitis' OR 'spondyloarthritis') AND ('non-steroidal anti-inflammatory drugs' OR 'etoricoxib' OR 'celecoxib' OR 'meloxicam' OR 'diclofenac' OR 'naproxen') AND 'randomized controlled trial'. The web-based search was supplemented with manual searches of references of relevant reviews on AS. When multiple studies describing the same population were published, the most complete study was used.

All double-blinded RCTs that enrolled patients fulfilling the modified 1984 AS New York criteria (23) were included and comparisons between different NSAIDs or of an NSAID with placebo were performed. The outcomes reported within 12 weeks were used. Studies were excluded if none of the quantitative outcomes of interest (see below) was reported. Studies on patients with concomitant treatment with prednisone $>10 \mathrm{mg} / \mathrm{d}$ or biologics were excluded, while those using sulfasalazine for patients who had been using them with stable doses prior to the study were considered.

Data extraction and quality assessment. Two researchers (MDF and JL) independently screened the studies retrieved for eligibility and extracted data from the trials included. The following data were extracted for each eligible RCT: First author, publication year, interventions, study duration, endpoints and patients' characteristics. Disagreements were resolved through discussion. Mean differences (MDs) and standard deviations were used to describe continuous outcomes and the number of events was used for dichotomous outcomes. When the values were not provided in the published article, the data were extracted from graphs. For trials that assessed more than one dose of an NSAID, the effects of different doses were pooled together. The present analysis was based on the intent-to-treat principle, which included all patients receiving at least one dose of the studied drug (20,22,24-27).

Evaluations of methodological quality of the RCTs included were performed independently by two reviewers (MDF and JL) according to the standard criteria of The Cochrane Collaboration (28), which consists of seven items: Random sequence generation, allocation concealment, blinding of participants, blinding of outcome assessment, incomplete outcome data, selective reporting and other bias. Regarding each of the above items, each study received a rating as low, high or unclear and two reviewers assessed each trial. The studies were then divided into three categories: i) Low risk of bias: Low risk of bias for all key domains; ii) moderate risk of bias: Unclear risk of bias for one or more key domains; iii) high risk of bias: High risk of bias for one or more key domains.

Endpoints of interest. The primary efficacy endpoints included the mean change in total pain score, patients' global assessment of disease activity (PGA) (29) and the Bath Ankylosing Spondylitis Functional Index (BASFI) (30). Pain and PGA scores were assessed using a 0-100 $\mathrm{mm}$ visual analog scale (VAS). BASFI assessment was performed using a series of 10 specific questions, each answered on a 0-100 mm VAS where 0 indicated 'easy' and 100 indicated 'impossible' (30). The secondary efficacy endpoints were the proportions of patients reaching the Assessment in Ankylosing Spondylitis 20 improvement criteria (ASAS20) (29), which were defined as an improvement of $\geq 20 \%$ and absolute improvement of $\geq 10$ units (0-100 mm VAS) from baseline in at least 3 of the following 4 domains: PGA, total back pain, BASFI and inflammation/morning stiffness, without any worsening of $\geq 20 \%$ and 10 units in the remaining domain.

The safety endpoints included total AEs, GI events, withdrawals due to AEs and serious AEs during the study. The GI events were defined as any abdominal complaints, including nausea, vomiting, dyspepsia, heartburn, diarrhea, constipation and abdominal pain.

Statistical analysis. Indirect comparisons were performed using a random-effects Bayesian network meta-analysis with WinBUGS version 1.4.3 (MRC Biostatistics Unit). Bayesian network meta-analysis incorporates direct and indirect comparisons of treatments, so as to derive estimates of effect of one treatment against another and perform a ranking of treatments (31-34).

For continuous data, the MDs were reported from the median of the posterior distribution with the accompanying 95\% credible intervals (CrIs). For dichotomous data, the odds ratios (ORs) with the $95 \%$ CrIs were presented. Furthermore, the probability of being the best $\left(\mathrm{P}_{\text {best }}\right)$ for each treatment was estimated (35). The goodness of model fit was assessed using the residual deviance to examine the validity of the network models, which should be close to the data points. To assess the robustness of the results, a sensitivity analysis was performed by including only trials with full daily doses of NSAIDs, i.e. etoricoxib $90 \mathrm{mg} / \mathrm{d}$, celecoxib $400 \mathrm{mg} / \mathrm{d}$, meloxicam $15 \mathrm{mg} / \mathrm{d}$, diclofenac $150 \mathrm{mg} / \mathrm{d}$, naproxen $1,000 \mathrm{mg} / \mathrm{d}$ and beta-D-mannuronic acid (M2000) 1,000 mg/d (36). 
A traditional meta-analysis was also performed using RevMan version 5.3.3 (Cochrane Collaboration) for the outcomes of withdrawals due to AEs and serious AEs. As these outcomes were rare events $(<10 \%)$, the Peto ORs with corresponding $95 \%$ confidence intervals (CIs) were calculated.

\section{Results}

Literature search. The selection process of trials for inclusion in the present study is summarized in Fig. 1. The literature research identified 1,806 records in total, 1,714 of which were excluded after screening their titles and abstracts. The full text of the 92 remaining, potentially eligible articles was reviewed. Finally, 9 RCTs (18-22,24-27) comprising 3,647 patients focusing on 6 NSAIDs, including etoricoxib, celecoxib, meloxicam, diclofenac, naproxen and M2000, were selected for analysis. All studies compared an NSAID with placebo or a different NSAID. A network diagram of treatment comparisons among these trials is provided in Fig. 2.

Characteristics of the included trials. The major characteristics of the RCTs included are summarized in Table I. While all of the patients included were diagnosed with AS, there were certain differences, e.g. in terms of the pain score, PGA and BASFI at baseline. The treatment duration varied from 6 to 12 weeks. All of the included studies were double-blinded RCTs. The results of the risk of bias assessment of the included trials are presented in Fig. 3. There was a moderate risk of bias in all trials, mostly due to lack of blinding of outcome assessment or allocation concealment.

Efficacy of NSAIDs. All of the 9 trials reported the mean change of the pain score (18-22,24-27). A total of 7 trials reported the PGA $(19,20,22,24-27), 7$ reported the BASFI $(18-20,22,24,25,27)$ and 6 reported the ASAS20 (18-20,22,24,27).

Compared with placebo, all NSAIDs were significantly more efficacious in reducing pain severity (MDs between -17.49 and -25.99 with a lower value indicating higher efficacy; Fig. 4A). Etoricoxib was significantly more effective than celecoxib in terms of pain alleviation $(\mathrm{MD}=-8.39$, $95 \%$ CrI: -16.55 to -0.79 ). Analysis of ranking probabilities indicated that etoricoxib had the highest probability of being the best treatment in decreasing pain severity $\left(\mathrm{P}_{\text {best }}, 73.8 \%\right)$ (Table II).

Similarly, significant improvements in PGA and BASFI were determined in patients receiving NSAIDs compared to placebo (Figs. 4B and 5A). Etoricoxib was superior to celecoxib in reducing the PGA score with statistical significance ( $\mathrm{MD}=-9.51,95 \% \mathrm{CrI}:-17.34$ to -1.45 ). However, there were no significant differences among the NSAIDs in decreasing the BASFI. All NSAIDs had a significantly higher rate of ASAS20 compared with placebo (ORs between 2.71 and 7.54; Fig. 5B). But celecoxib was significantly less efficacious in reaching ASAS20 than etoricoxib (OR=0.36, 95\% CrI: 0.15-0.85). The probability analysis suggested that etoricoxib remained the most effective option for the outcomes of PGA, BASFI and ASAS20 ( $\mathrm{P}_{\text {best }}$ of 67.2, 76.1 and 71.8\%, respectively; Table II).

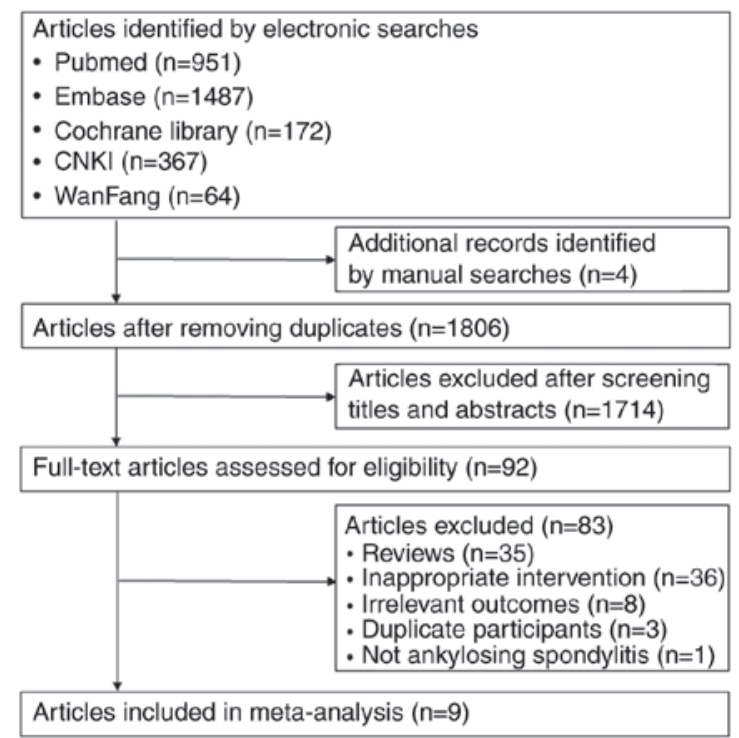

Figure 1. Flowchart of the study selection process.

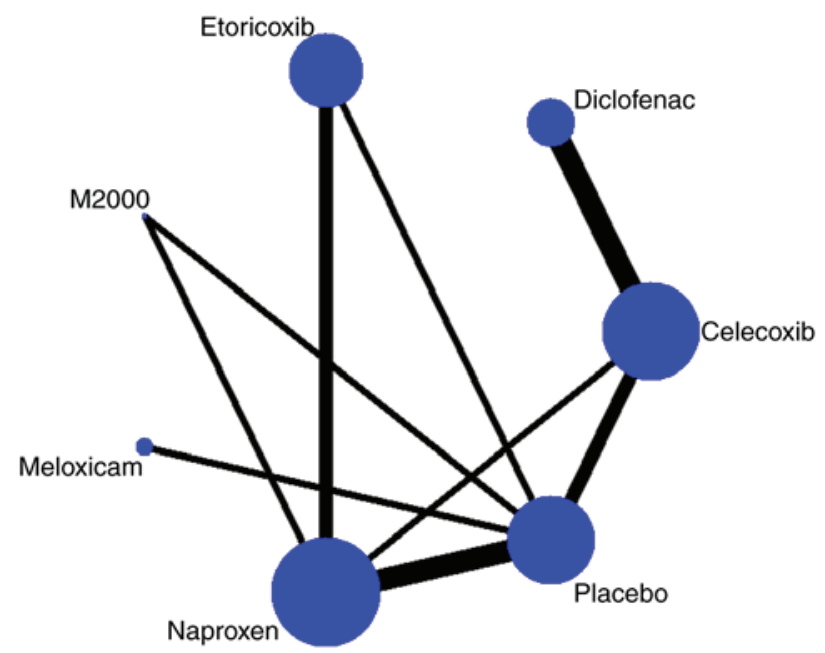

Figure 2. Network diagram of the comparisons in the meta-analysis. The size of the nodes is proportionate to the number of patients randomized to the treatment. The width of the lines is proportionate to the number of direct comparisons between the connected treatments.

The test for goodness of model fit suggested that the models of efficacy outcomes were appropriate. In the sensitivity analysis of full-dose NSAIDs trials, all NSAIDs were highly effective in improving pain, PGA, BASFI and achieving the ASAS20, except that diclofenac had an inconclusive higher rate of ASAS20 than placebo (data not shown). Etoricoxib remained the best therapy regarding the outcomes of pain, PGA, BASFI and ASAS20 ( $\mathrm{P}_{\text {best }}$ of 77.8, 65.6, 66.5 and $67.1 \%$, respectively). However, no significant differences were obtained between etoricoxib and celecoxib in improving pain, PGA scores and ASAS20.

Safety of NSAIDs. A total of 8 RCTs reported on the occurrence of total AEs, GI events, withdrawals due to AEs and serious AEs (18-22,24,25,27). Meloxicam was not included in the analysis due to a lack of data. 


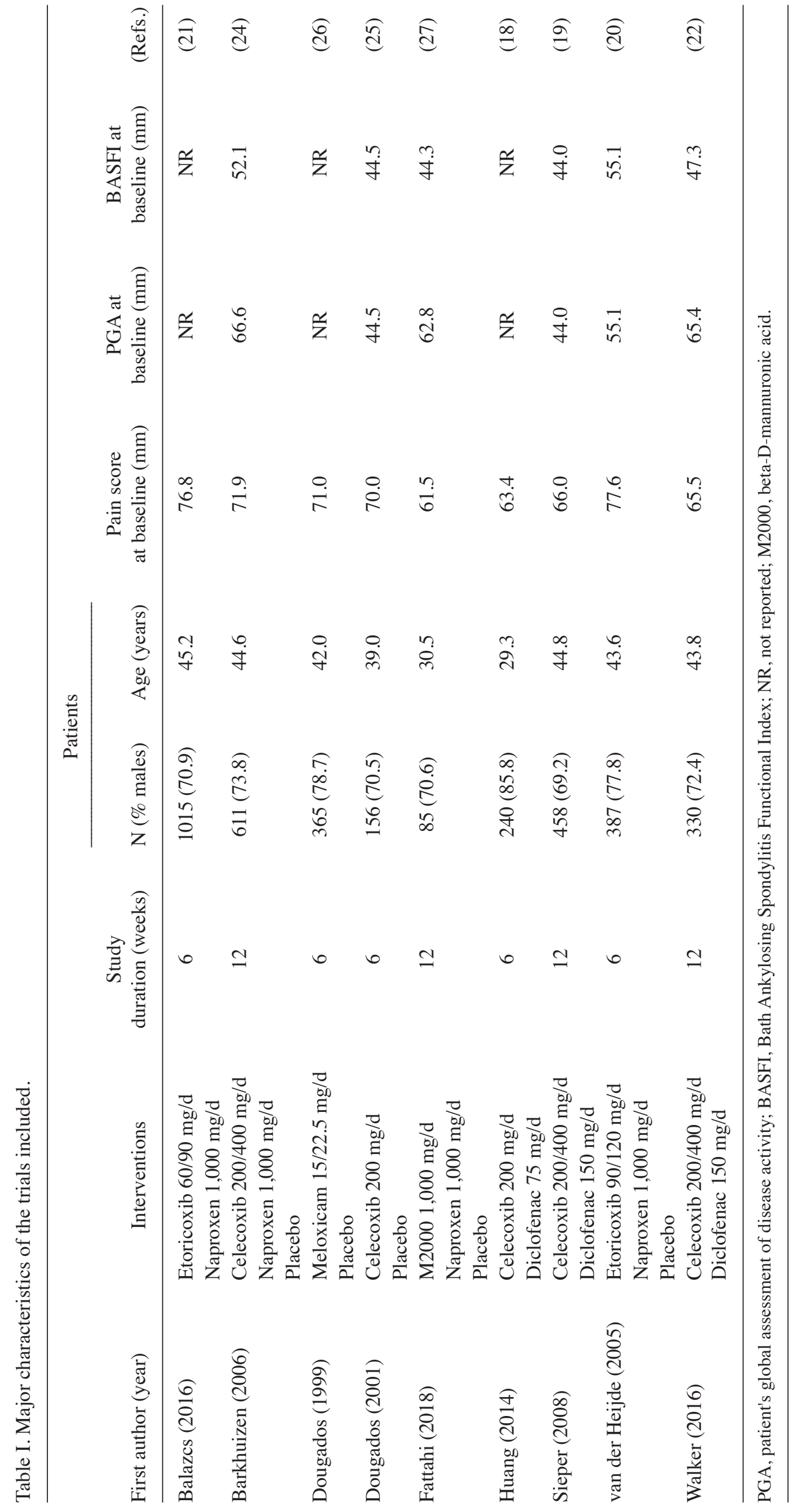


Table II. Determination of the most effective non-steroidal anti-inflammatory drug in the treatment of ankylosing spondylitis.

\begin{tabular}{lcccc}
\hline Agent & Change in pain score $(\%)$ & Change in PGA score $(\%)$ & Change in BASFI score (\%) & ASAS20 (\%) \\
\hline Etoricoxib & 73.8 & 67.2 & 76.1 & 0.58 \\
Celecoxib & 0.19 & 0.00 & NA & 0.22 \\
Meloxicam & 4.62 & 25.1 & 3.54 & NA \\
Diclofenac & 4.28 & 2.06 & 12.1 & 1.53 \\
Naproxen & 11.8 & 2.10 & 7.70 & 2.62 \\
M2000 & 5.33 & 3.50 & & 23.8
\end{tabular}

PGA, patient's global assessment of disease activity; BASFI, Bath Ankylosing Spondylitis Functional Index; ASAS20, Assessment in Ankylosing Spondylitis 20\% response; NA, not available; M2000, beta-D-mannuronic acid.

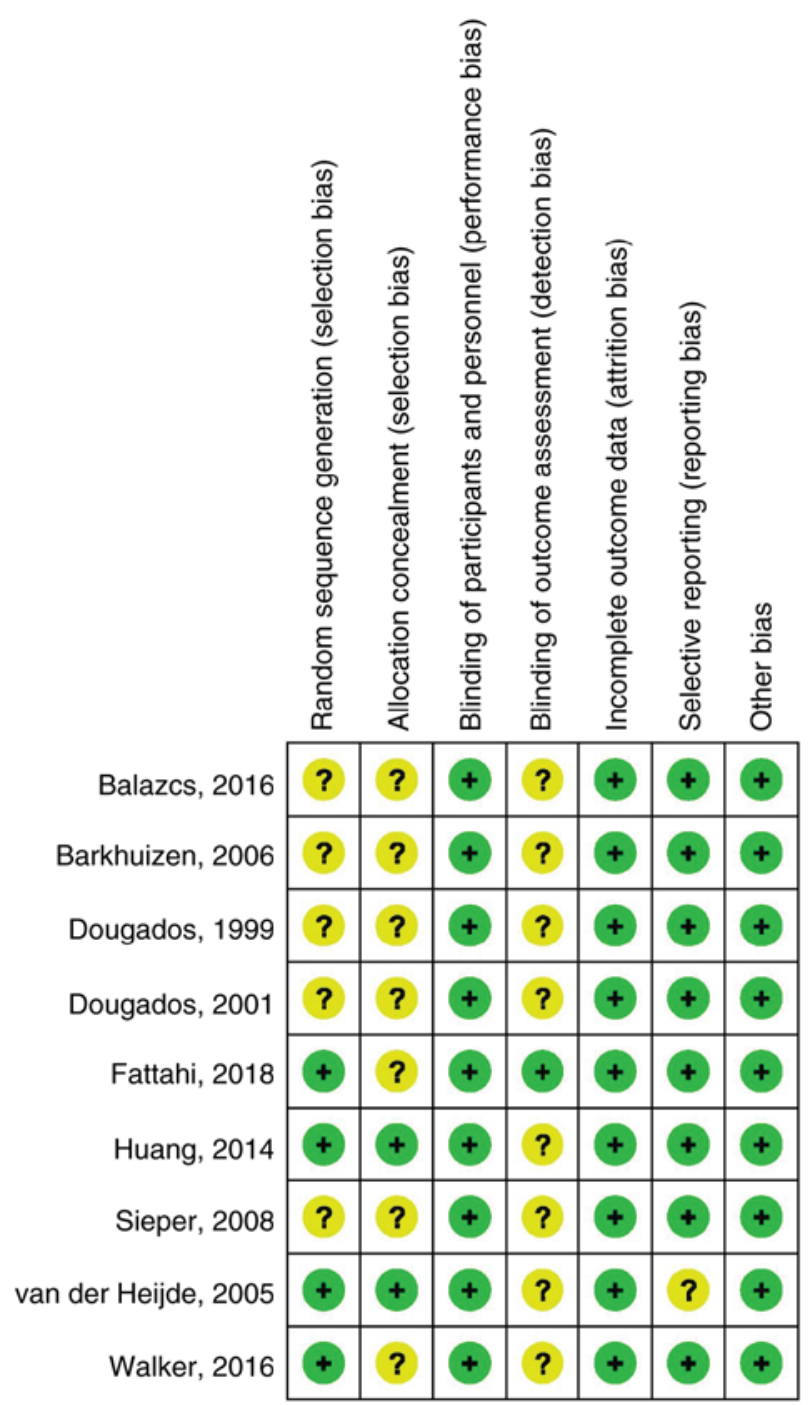

Figure 3. Assessment of the risk of bias for the studies included. Question marks indicate unclear risk of bias and plus symbols indicate low risk of bias.

Network meta-analysis demonstrated no statistically significant differences among NSAIDs and placebo regarding the risk of total AEs (Fig. 6A). Additionally, no significant differences were identified in AEs of the M2000 and placebo (OR=0.39, 95\% CrI: 0.10-1.43). The probability analysis indicated that M2000 had the greatest probability of being the safest treatment $\left(\mathrm{P}_{\text {best }}, 90.5 \%\right)$. Furthermore, M2000 was associated with a lower incidence of AEs than celecoxib and diclofenac $(\mathrm{OR}=0.26,95 \% \mathrm{CrI}: 0.06-1.00$ and $\mathrm{OR}=0.23,95 \%$ CrI: 0.05-0.99, respectively).

Regarding the risk of GI events, patients treated with diclofenac and naproxen had a significantly higher risk than those taking placebo $(\mathrm{OR}=2.87,95 \% \mathrm{CrI}: 1.06-7.67$ and $\mathrm{OR}=2.38$, 95\% CrI: 1.08-4.93, respectively; Fig. 6B). No significant differences in terms of GI events were determined among the different NSAIDs. However, M2000 had a considerable probability of being ranked as the safest drug for decreasing GI events (62.2\%). Regarding withdrawals due to AEs and serious AEs, there were no statistically significant differences among NSAIDs and placebo (Fig. 7A and B).

The test for goodness of model fit suggested that the models of safety endpoints were appropriate. In the sensitivity analysis of full-dose trials, no significant differences in total AEs were identified among NSAIDs and placebo groups. Additionally, no significant differences were identified in the GI events for diclofenac and naproxen when compared with the placebo. M2000 remained the safest therapy for the outcomes of AEs and GI events $\left(\mathrm{P}_{\text {best }}\right.$ of 87.3 and $61.4 \%$, respectively). The results of withdrawals due to AEs and serious AEs remained stable during sensitivity analysis.

\section{Discussion}

The present meta-analysis provides comparative information on the efficacy and safety of commonly used NSAIDs in the treatment of AS. The analysis confirmed that NSAIDs were consistently more effective than placebo in improving pain, disease activity and physical function in patients with AS. Furthermore, etoricoxib was significantly superior to celecoxib in reducing pain, PGA scores and achievement of ASAS20. However, in the sensitivity analysis of full-dose NSAID trials, no significant differences in efficacy outcomes were obtained between etoricoxib and celecoxib. However, etoricoxib still ranked as the most efficacious treatment for AS.

Likewise, the trials comparing etoricoxib to naproxen directly identified that etoricoxib had superior or comparable efficacy compared with naproxen $(20,21)$. In their recent indirect comparison of NSAIDs for AS, Wang et al (37) also demonstrated that etoricoxib was more effective than certain 
A

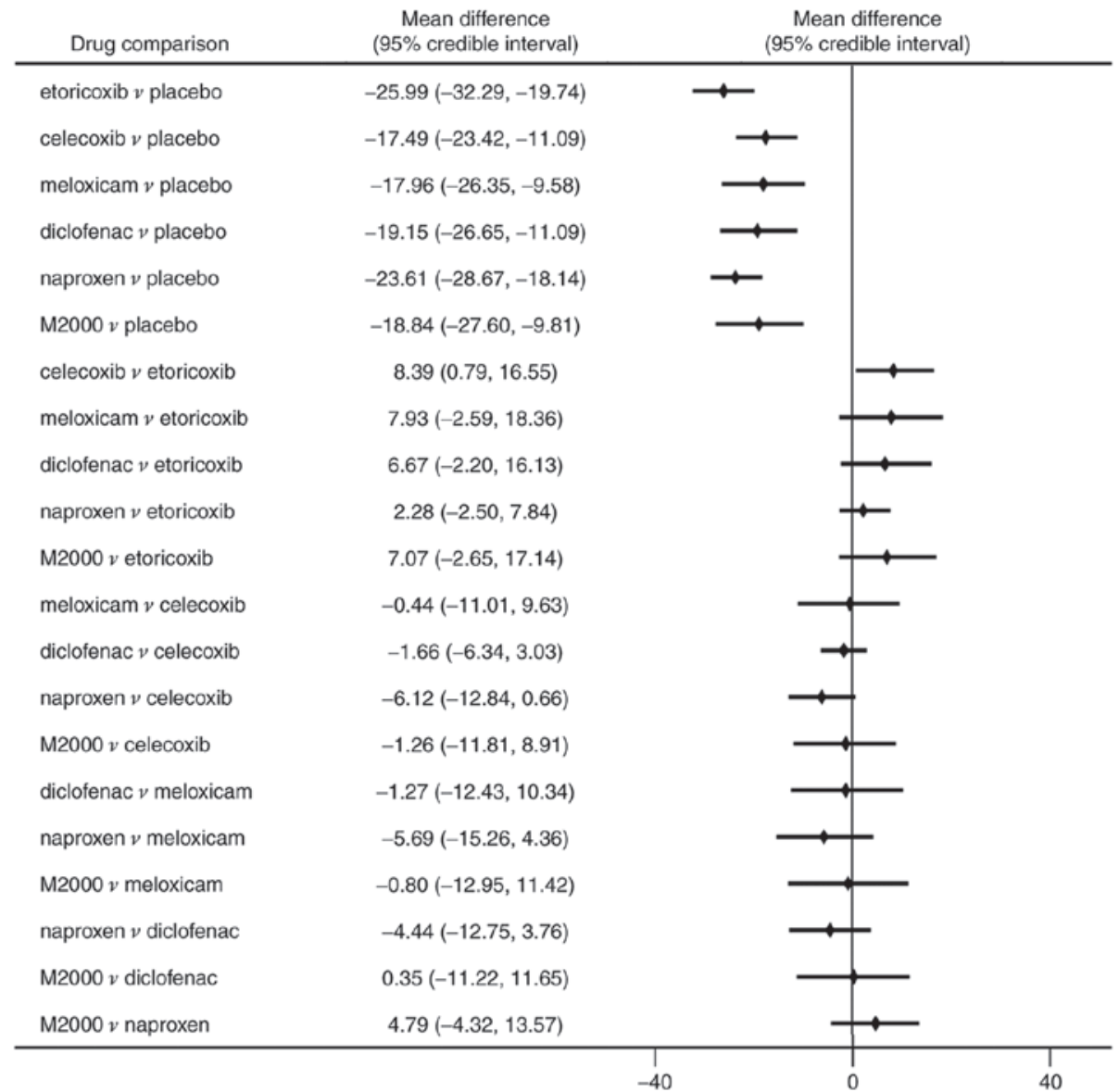

B

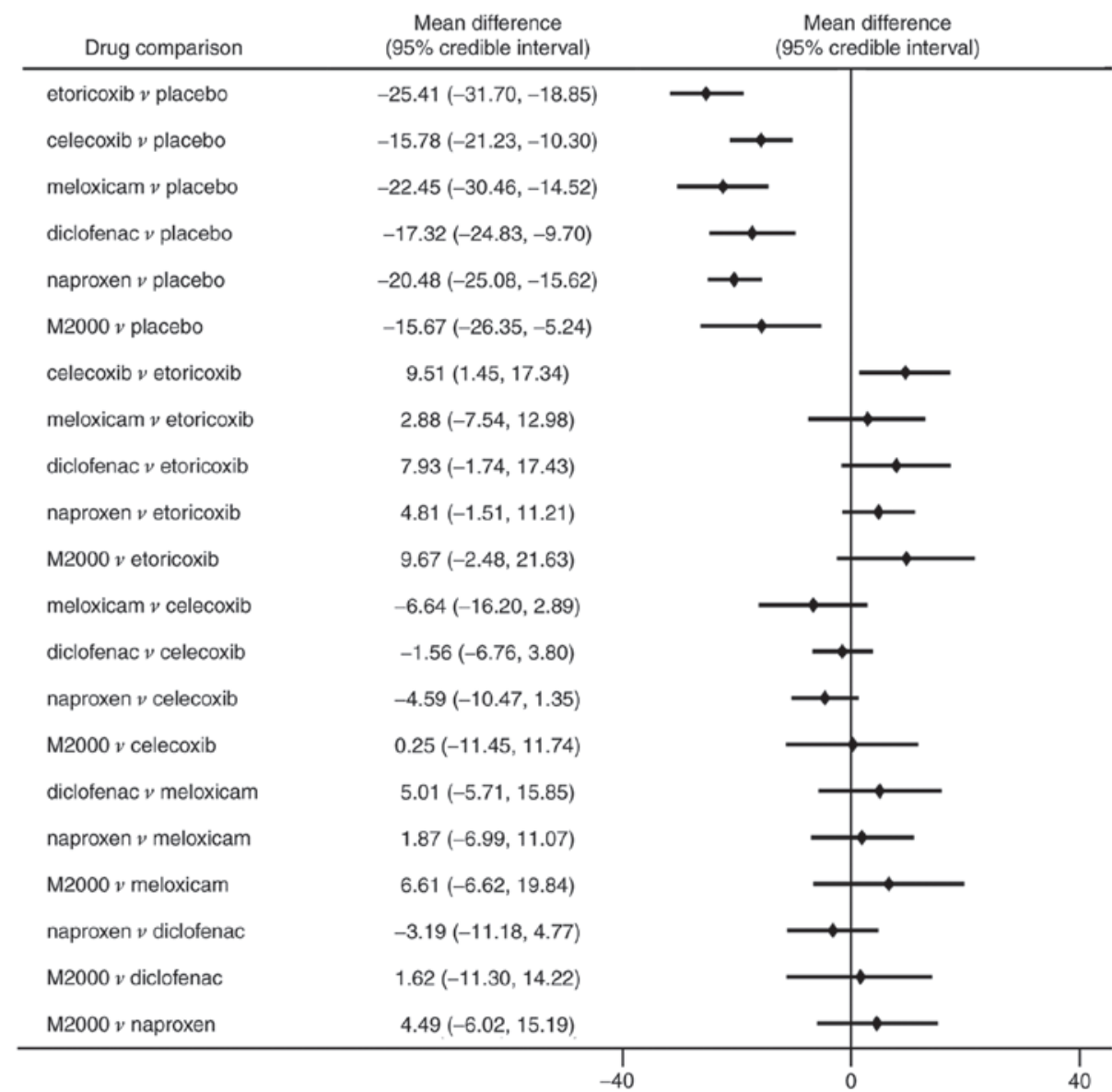

Figure 4. Efficacy endpoints of NSAIDs in ankylosing spondylitis. MDs among NSAIDs and placebo for (A) pain score and (B) patient's global assessment of disease activity. A negative MD value indicates a benefit from therapy. MD, mean difference; NSAIDs, non-steroidal anti-inflammatory drugs. 


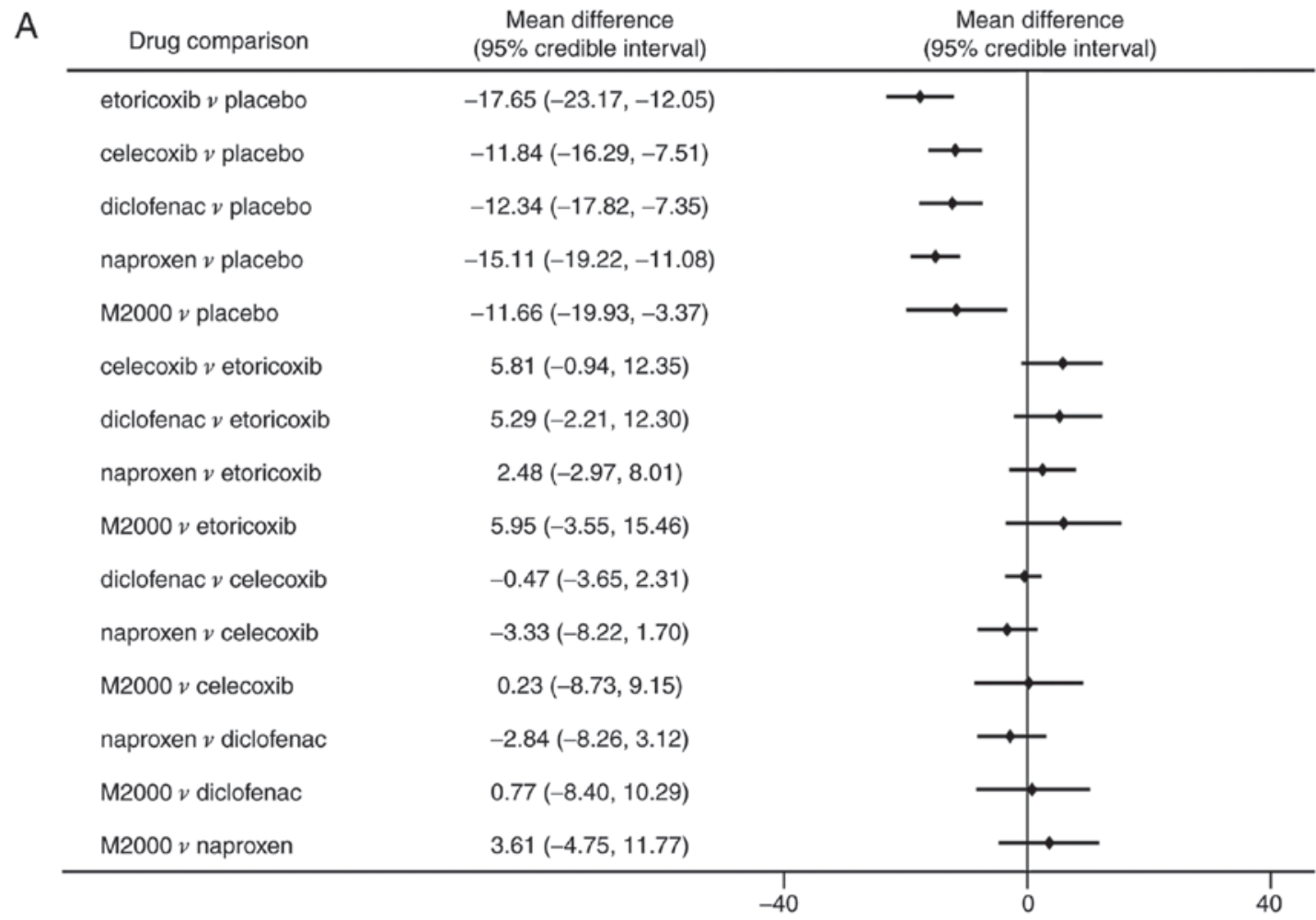

\begin{tabular}{lcl} 
Drug comparison & $\begin{array}{c}\text { Odds ratio } \\
(95 \% \text { credible interval })\end{array}$ \\
\hline etoricoxib $v$ placebo & $7.54(3.83,15.31)$ \\
celecoxib $v$ placebo & $2.71(1.44,5.22)$ \\
diclofenac $v$ placebo & $3.14(1.49,6.82)$ \\
naproxen $v$ placebo & $4.64(2.88,7.82)$ \\
M2000 $v$ placebo & $5.02(1.68,15.29)$ \\
celecoxib $v$ etoricoxib & $0.36(0.15,0.85)$ \\
diclofenac $v$ etoricoxib & $0.42(0.16,1.08)$ \\
naproxen $v$ etoricoxib & $0.61(0.32,1.22)$ \\
M2000 $v$ etoricoxib & $0.66(0.19,2.32)$ \\
diclofenac $v$ celecoxib & $1.16(0.77,1.75)$ \\
naproxen $v$ celecoxib & $1.71(0.92,3.25)$ \\
M2000 $v$ celecoxib & $1.85(0.57,6.12)$ \\
naproxen $v$ diclofenac & $1.48(0.70,3.14)$ \\
M2000 $v$ diclofenac & $1.59(0.46,5.68)$ \\
M2000 $v$ naproxen & $1.08(0.37,3.19)$ \\
\hline
\end{tabular}

Figure 5. Efficacy endpoints of NSAIDs in ankylosing spondylitis. (A) MDs among NSAIDs and the placebo for Bath Ankylosing Spondylitis Functional Index. (B) ORs among NSAIDs and the placebo for assessment of the Ankylosing Spondylitis $20 \%$ response. A negative MD value or an OR of $>1.0$ indicated a benefit from therapy. MD, mean difference; NSAIDs, non-steroidal anti-inflammatory drugs; OR, odds ratio.

other NSAIDs in reducing pain, which is in accordance with the present results. Furthermore, an economic evaluation indicated that etoricoxib was a more cost-effective treatment for AS compared to celecoxib, diclofenac and naproxen for a duration of $>5$ years (38).
With regard to safety, there were no significant differences between NSAIDs and placebo in terms of total AEs, withdrawals due to AEs or serious AEs. Additionally, no significant differences in AEs were identified between M2000 and the placebo. However, M2000 was ranked as the 
A

Drug comparison

Odds ratio

Odds ratio (95\% credible interval) (95\% credible interval)

\begin{tabular}{ll}
\hline etoricoxib $v$ placebo & $1.33(0.68,2.74)$ \\
celecoxib $v$ placebo & $1.50(0.88,3.04)$ \\
diclofenac $v$ placebo & $1.71(0.80,4.05)$ \\
naproxen $v$ placebo & $1.17(0.70,2.08)$ \\
M2000 $v$ placebo & $0.39(0.10,1.43)$ \\
celecoxib $v$ etoricoxib & $1.13(0.51,2.81)$ \\
diclofenac $v$ etoricoxib & $1.29(0.48,3.59)$ \\
naproxen $v$ etoricoxib & $0.89(0.49,1.59)$ \\
M2000 $v$ etoricoxib & $0.29(0.06,1.15)$ \\
diclofenac $v$ celecoxib & $1.14(0.65,1.89)$ \\
naproxen $v$ celecoxib & $0.78(0.37,1.48)$ \\
M2000 $v$ celecoxib & $0.26(0.06,1.00)$ \\
naproxen $v$ diclofenac & $0.69(0.28,1.61)$ \\
M2000 $v$ diclofenac & $0.23(0.05,0.99)$ \\
M2000 $v$ naproxen & $0.33(0.08,1.18)$ \\
\hline
\end{tabular}

B

\begin{tabular}{lcl|l|} 
Drug comparison & $\begin{array}{c}\text { Odds ratio } \\
(95 \% \text { credible interval })\end{array}$ \\
\hline etoricoxib $v$ placebo & $2.58(0.95,6.98)$ \\
celecoxib $v$ placebo & $1.71(0.81,3.70)$ \\
diclofenac $v$ placebo & $2.87(1.06,7.67)$ \\
naproxen $v$ placebo & $2.38(1.08,4.93)$ \\
M2000 $v$ placebo & $0.73(0.08,4.47)$ \\
celecoxib $v$ etoricoxib & $0.66(0.22,2.07)$ \\
diclofenac $v$ etoricoxib & $1.11(0.31,4.06)$ \\
naproxen $v$ etoricoxib & $0.92(0.40,2.01)$ \\
M2000 $v$ etoricoxib & $0.28(0.03,1.96)$ \\
diclofenac $v$ celecoxib & $1.68(0.86,3.13)$ \\
naproxen $v$ celecoxib & $1.39(0.56,3.12)$ \\
M2000 $v$ celecoxib & $0.42(0.04,2.83)$ & \\
naproxen $v$ diclofenac & $0.83(0.27,2.36)$ & \\
M2000 $v$ diclofenac & $0.25(0.02,1.89)$ & \\
M2000 $v$ naproxen & $0.30(0.04,1.84)$ & \\
\hline
\end{tabular}

Figure 6. Safety endpoints of NSAIDs in ankylosing spondylitis. ORs among NSAIDs and placebo for (A) AEs and (B) gastrointestinal AEs. An OR of <1.0 indicates a benefit from therapy. AE, adverse event; NSAIDs, non-steroidal anti-inflammatory drugs; OR, odds ratio.

safest drug for AEs and GI events. M2000, a novel NSAID with immunosuppressive properties, has been indicated to be well tolerated with a high safety profile for the digestive system and kidney (39). In a recent RCT, Fattahi et al demonstrated that M2000 had similar efficacy, but lower risk of GI and other AEs than naproxen for the treatment of AS (27). 
A

\begin{tabular}{|c|c|c|}
\hline Drug comparison & $\begin{array}{l}\text { Peto odds ratio } \\
\text { (95\% Confidence interval) }\end{array}$ & $\begin{array}{c}\text { Peto odds ratio } \\
\text { (95\% Confidence interval) }\end{array}$ \\
\hline Etoricoxib $v$ placebo & $4.40(0.23,85.73)$ & \\
\hline Celecoxib $v$ placebo & $0.90(0.41,1.98)$ & - \\
\hline Naproxen $v$ placebo & $1.18(0.52,2.68)$ & \\
\hline Naproxen $v$ etoricoxib & $1.36(0.54,3.44)$ & \\
\hline Diclofenac $v$ celecoxib & $1.45(0.91,2.31)$ & - \\
\hline Naproxen $v$ celecoxib & $1.47(0.59,3.69)$ & \\
\hline M2000 $v$ naproxen & $0.12(0.01,1.17)$ & \\
\hline M2000 $v$ placebo & Not estimable & \\
\hline
\end{tabular}

B

\begin{tabular}{lcc|c} 
Drug comparison & $\begin{array}{c}\text { Peto odds ratio } \\
(95 \% \text { Confidence interval })\end{array}$ & $\begin{array}{c}\text { Peto odds ratio } \\
(95 \% \text { Confidence interval })\end{array}$ \\
\hline Etoricoxib $v$ placebo & $4.38(0.07,289.6)$ & & \\
Celecoxib $v$ placebo & $0.89(0.14,5.53)$ & & \\
Naproxen $v$ placebo & $1.49(0.26,8.70)$ & & \\
Naproxen $v$ etoricoxib & $0.28(0.04,2.01)$ & & \\
Diclofenac $v$ celecoxib & $1.01(0.52,1.97)$ & & \\
Naproxen $v$ celecoxib & $3.12(0.49,19.89)$ & & \\
M2000 $v$ naproxen & Not estimable & & 1 \\
M2000 $v$ placebo & Not estimable & & 1 \\
\hline
\end{tabular}

Figure 7. Safety endpoints of NSAIDs in ankylosing spondylitis. ORs among NSAIDs and the placebo for (A) withdrawals due to AEs and (B) serious AEs. An OR of <1.0 indicated a benefit from therapy. AE, adverse event; NSAIDs, non-steroidal anti-inflammatory drugs; OR, odds ratio.

In addition, patients taking diclofenac or naproxen complained of more GI events than those with placebo. Thus, clinicians should take the risk of GI events into account when prescribing NSAIDs. In the present analysis, there were no significant differences in safety among etoricoxib, celecoxib and non-selective NSAIDs. The reason may be that AS patients are on average young and, therefore, have a lower risk of GI events. These results are consistent with those of a recent cohort study, which identified no significantly increased risks of GI and cardiovascular events for etoricoxib, celecoxib and non-selective NSAIDs (40).

The 2016 update of the Assessment of SpondyloArthritis International Society/European League Against Rheumatism recommendations for managing AS suggests that NSAIDs are the mainstay of treatment for patients with AS (8). Several studies have indicated that continuous use of NSAIDs may slow radiographic progression in symptomatic patients, as assessed by the modified Stoke Ankylosing Spondylitis spinal score $(10,11,41)$. Conversely, a recent study demonstrated that continuous treatment with diclofenac over 2 years was not able to reduce radiographic progression compared with on-demand treatment (42). There is an ongoing debate regarding whether NSAIDs are effective in inhibiting new bone formation (1). To date, the available data are too scant to draw any conclusions for clinical practice. Further long-term prospective studies should be performed.

Of note, the present study has several limitations. Firstly, the literature review only identified a limited number of the RCTs available, and meloxicam and M2000 were studied in only one trial, which may affect the robustness of the comparison. Secondly, different doses of an NSAID were pooled together, which may have introduced certain heterogeneity into the analysis. However, in the sensitivity analysis of full-dose trials, the results were not markedly affected. In addition, there were certain variations in the pain score, PGA, BASFI at baseline and treatment duration among trials, which may have affected the efficacy of NSAIDs. Furthermore, NSAIDs were not assessed in patients with 
non-radiographic axial spondyloarthritis due to the paucity of data. Finally, only the short-term efficacy and safety of NSAIDs were investigated. Further studies with adequate follow-up are required to assess the long-term efficacy and safety of NSAIDs in patients with AS.

In summary, NSAIDs are all highly effective and well-tolerated compared to placebo in the treatment of AS. Clinicians should take GI toxicity into account when prescribing NSAIDs.

\section{Acknowledgements}

The results of this study were previously presented at the 18th Asia Pacific League of Associations for Rheumatology Congress in Shanghai (China) in 2016 (abstract no. APL16-0754).

\section{Funding}

This work was supported by the National Science Foundation of China (grant nos. 81871294, 81972204 and 81702327), the Natural Science Foundation of Guangdong Province (grant no. 2019A1515011097), the President Foundation of Nanfang Hospital, Southern Medical University (grant no. 2016C010), the China Postdoctoral Science Foundation (grant nos. 2018M640834 and 2019T120756), the Science and Technology Planning Project of Guangzhou (grant no. 201904010089), Innovation Program of Shenzhen (grant no. JCYJ20180508165208399) and the 111 Project from the Ministry of Education of China (grant no. D18010).

\section{Availability of data and materials}

The datasets used and/or analyzed during the present study are available from the corresponding author on reasonable request.

\section{Authors' contributions}

JG conceived the study. JG, XL, MF, JL and XW designed the study. MF and JL performed the literature search and data extraction. MF and BZ performed the network meta-analysis. JG, XL, MF, JL and XW interpreted the data. MF and JL wrote the first draft of the manuscript. XL and JG critically revised the manuscript and provided final approval of the manuscript.

\section{Ethics approval and consent to participate}

Not applicable.

\section{Patient consent for publication}

Not applicable.

\section{Competing interests}

The authors declare that they have no competing interests.

\section{References}

1. Sieper J and Poddubnyy D: Axial spondyloarthritis. Lancet 390: 73-84, 2017.
2. Braun J and Sieper J: Ankylosing spondylitis. Lancet 369: 1379-1390, 2007.

3. Guan M, Wang J,Zhao L, Xiao J, Li Z and Shi Z: Management of hip involvement in ankylosing spondylitis. Clin Rheumatol 32: 1115-1120, 2013.

4. Wang J, Zhang Y, Zhao L, Li ZH and Shi ZJ: The efficacy and safety of infliximab used in patients with ankylosing spondylitis after unilateral total hip arthroplasty. Hip Int 23: 406-410, 2013.

5. Guan M, Wang J, Zhu Z, Xiao J, Zhao L, Li Z and Shi Z: Comparison in clinical features and life impact between juvenile-onset and adult-onset ankylosing spondylitis. Turk J Med Sci 44: 601-605, 2014.

6. Boonen A, Chorus A, Miedema H, van der Heijde D, van der Tempel $\mathrm{H}$ and van der Linden S: Employment, work disability, and work days lost in patients with ankylosing spondylitis: A cross sectional study of Dutch patients. Ann Rheum Dis 60: 353-358, 2001.

7. Ward MM, Deodhar A, Akl EA, Lui A, Ermann J, Gensler LS, Smith JA, Borenstein D, Hiratzka J, Weiss PF, Inman RD, et al: American College of Rheumatology/Spondylitis Association of America/Spondyloarthritis Research and Treatment Network 2015 recommendations for the treatment of ankylosing spondylitis and nonradiographic axial spondyloarthritis. Arthritis Care Res (Hoboken) 68: 151-166, 2016.

8. Braun J, van den Berg $\mathrm{R}$, Baraliakos $\mathrm{X}$, Boehm $\mathrm{H}$, Burgos-Vargas R, Collantes-Estevez E, Dagfinrud H, Dijkmans B, Dougados M, Emery P, et al: 2010 update of the ASAS/EULAR recommendations for the management of ankylosing spondylitis. Ann Rheum Dis 70: 896-904, 2011.

9. Zochling J, van der Heijde D, Burgos-Vargas R, Collantes E, Davis JC, Jr., Dijkmans B, Dougados M, Géher P, Inman RD, Khan MA, et al: ASAS/EULAR recommendations for the management of ankylosing spondylitis. Ann Rheum Dis 65: 442-452, 2006.

10. Poddubnyy D, Rudwaleit M,Haibel H,Listing J, Märker-HermannE, Zeidler H, Braun J and Sieper J: Effect of non-steroidal anti-inflammatory drugs on radiographic spinal progression in patients with axial spondyloarthritis: Results from the German Spondyloarthritis Inception Cohort. Ann Rheum Dis 71: 1616-1622, 2012.

11. Wanders A, Heijde D, Landewé R, Béhier JM, Calin A, Olivieri I, Zeidler $\mathrm{H}$ and Dougados $\mathrm{M}$ : Nonsteroidal antiinflammatory drugs reduce radiographic progression in patients with ankylosing spondylitis: A randomized clinical trial. Arthritis Rheum 52: 1756-1765, 2005.

12. Shukla A, Rai MK, Prasad N and Agarwal V: Short-term non-steroid anti-inflammatory drug use in spondyloarthritis patients induces subclinical acute kidney injury: Biomarkers study. Nephron 135: 277-286, 2017.

13. Song IH, Poddubnyy DA, Rudwaleit M and Sieper J: Benefits and risks of ankylosing spondylitis treatment with nonsteroidal antiinflammatory drugs. Arthritis Rheum 58: 929-938, 2008.

14. Fosbøl EL, Køber L, Torp-Pedersen C and Gislason GH: Cardiovascular safety of non-steroidal anti-inflammatory drugs among healthy individuals. Expert Opin Drug Saf 9: 893-903, 2010.

15. Combe B, Swergold G, McLay J, McCarthy T, Zerbini C, Emery P, Connors L, Kaur A, Curtis S, Laine L and Cannon CP: Cardiovascular safety and gastrointestinal tolerability of etoricoxib vs diclofenac in a randomized controlled clinical trial (The MEDAL study). Rheumatology (Oxford) 48: 425-432, 2009.

16. Rostom A, Muir K, Dubé C, Jolicoeur E, Boucher M, Joyce J, Tugwell P and Wells GW: Gastrointestinal safety of cyclooxygenase-2 inhibitors: A Cochrane Collaboration systematic review. Clin Gastroenterol Hepatol 5: 818-828, 828.e1-e5; quiz $768,2007$.

17. Trelle S, Reichenbach S, Wandel S, Hildebrand P, Tschannen B, Villiger PM, Egger M and Jüni P: Cardiovascular safety of non-steroidal anti-inflammatory drugs: Network meta-analysis. BMJ 342: c7086, 2011.

18. Huang F, Gu J, Liu Y, Zhu P, Zheng Y, Fu J, Pan S and Le S: Efficacy and safety of celecoxib in chinese patients with ankylosing spondylitis: A 6-week randomized, double-blinded study with 6-week open-label extension treatment. Curr Ther Res Clin Exp 76: 126-133, 2014.

19. Sieper J, Klopsch T, Richter M, Kapelle A, Rudwaleit M, Schwank S, Regourd E and May M: Comparison of two different dosages of celecoxib with diclofenac for the treatment of active ankylosing spondylitis: Results of a 12-week randomised, double-blind, controlled study. Ann Rheum Dis 67: 323-329, 2008. 
20. van der Heijde D, Baraf HS, Ramos-Remus C, Calin A, Weaver AL, Schiff M, James M, Markind JE, Reicin AS, Melian A and Dougados M: Evaluation of the efficacy of etoricoxib in ankylosing spondylitis: Results of a fifty-two-week, randomized, controlled study. Arthritis Rheum 52: 1205-1215, 2005.

21. Balazcs E, Sieper J, Bickham K, Mehta A, Frontera N, Stryszak P, Popmihajlov Z and Peloso PM: A randomized, clinical trial to assess the relative efficacy and tolerability of two doses of etoricoxib versus naproxen in patients with ankylosing spondylitis. BMC Musculoskelet Disord 17: 426, 2016.

22. Walker C, Essex MN, Li C and Park PW: Celecoxib versus diclofenac for the treatment of ankylosing spondylitis: 12-week randomized study in Norwegian patients. J Int Med Res 44: 483-495, 2016

23. van der Linden S, Valkenburg HA and Cats A: Evaluation of diagnostic criteria for ankylosing spondylitis. A proposal for modification of the New York criteria. Arthritis Rheum 27: 361-368, 1984

24. Barkhuizen A, Steinfeld S, Robbins J, West C, Coombs J and Zwillich S: Celecoxib is efficacious and well tolerated in treating signs and symptoms of ankylosing spondylitis. J Rheumatol 33: 1805-1812, 2006.

25. Dougados M, Béhier JM, Jolchine I, Calin A, van der Heijde D, Olivieri I, Zeidler H and Herman H: Efficacy of celecoxib, a cyclooxygenase 2-specific inhibitor, in the treatment of ankylosing spondylitis: A six-week controlled study with comparison against placebo and against a conventional nonsteroidal antiinflammatory drug. Arthritis Rheum 44: 180-185, 2001.

26. Dougados M, Gueguen A, Nakache JP, Velicitat P, Veys EM, Zeidler $\mathrm{H}$ and Calin A: Ankylosing spondylitis: What is the optimum duration of a clinical study? A one year versus a 6 weeks non-steroidal anti-inflammatory drug trial. Rheumatology (Oxford) 38: 235-244, 1999.

27. Fattahi MJ, Jamshidi AR, Mahmoudi M, Vojdanian M, Yekaninejad MS, Jafarnezhad-Ansariha F, Ahmadi H, Rehm BHA, Matsuo H, Cuzzocrea S, et al: Evaluation of the efficacy and safety of beta-d-mannuronic acid in patients with ankylosing spondylitis: A 12-week randomized, placebo-controlled, phase I/II clinical trial. Int Immunopharmacol 54: 112-117, 2018.

28. Higgins J and Green S: Cochrane handbook of systematic reviews of interventions. Version 5.1.0 Cochrane Collaboration, 2008. Available: http://www.cochrane-handbook.org.

29. Calin A, Garrett S, Whitelock H, Kennedy LG, O'Hea J, Mallorie P and Jenkinson T: A new approach to defining functional ability in ankylosing spondylitis: The development of the Bath Ankylosing Spondylitis Functional Index. J Rheumatol 21: 2281-2285, 1994.

30. Anderson JJ, Baron G, van der Heijde D, Felson DT and Dougados M: Ankylosing spondylitis assessment group preliminary definition of short-term improvement in ankylosing spondylitis. Arthritis Rheum 44: 1876-1886, 2001.

31. Lu G and Ades AE: Combination of direct and indirect evidence in mixed treatment comparisons. Stat Med 23: 3105-3124, 2004
32. Sutton AJ and Abrams KR: Bayesian methods in meta-analysis and evidence synthesis. Stat Methods Med Res 10: 277-303, 2001.

33. Caldwell DM, Ades AE and Higgins JP: Simultaneous comparison of multiple treatments: Combining direct and indirect evidence. BMJ 331: 897-900, 2005

34. Zhao BC, Huang TY, Deng QW, Liu WF, Liu J, Deng WT, Liu KX and Li C: Prophylaxis against atrial fibrillation after general thoracic surgery: Trial sequential analysis and network meta-analysis. Chest 151: 149-159, 2017.

35. Salanti G, Ades AE and Ioannidis JP: Graphical methods and numerical summaries for presenting results from multiple-treatment meta-analysis: An overview and tutorial. J Clin Epidemiol 64: 163-171, 2011.

36. Dougados M, Simon P, Braun J, Burgos-Vargas R, Maksymowych WP, Sieper J and van der Heijde D: ASAS recommendations for collecting, analysing and reporting NSAID intake in clinical trials/epidemiological studies in axial spondyloarthritis. Ann Rheum Dis 70: 249-251, 2011.

37. Wang R, Dasgupta A and Ward MM: Comparative efficacy of non-steroidal anti-inflammatory drugs in ankylosing spondylitis: A Bayesian network meta-analysis of clinical trials. Ann Rheum Dis 75: 1152-1160, 2016.

38. Jansen JP and Taylor SD: Cost-effectiveness evaluation of etoricoxib versus celecoxib and nonselective NSAIDs in the treatment of ankylosing spondylitis in Norway. Int J Rheumatol 2011: 160326, 2011.

39. Fattahi MJ, Abdollahi M, Agha Mohammadi A, Rastkari N, Khorasani R, Ahmadi H, Tofighi Zavareh F, Sedaghat R, Tabrizian N and Mirshafiey A: Preclinical assessment of $\beta$-D-mannuronic acid (M2000) as a nonsteroidal anti-inflammatory drug. Immunopharmacol Immunotoxicol 37: 535-540, 2015.

40. Kristensen LE, Jakobsen AK, Askling J, Nilsson F and Jacobsson LT: Safety of etoricoxib, celecoxib, and nonselective nonsteroidal antiinflammatory drugs in ankylosing spondylitis and other spondyloarthritis patients: A Swedish National Population-Based Cohort study. Arthritis Care Res (Hoboken) 67: 1137-1149, 2015.

41. Kroon F, Landewé R, Dougados M and van der Heijde D: Continuous NSAID use reverts the effects of inflammation on radiographic progression in patients with ankylosing spondylitis. Ann Rheum Dis 71: 1623-1629, 2012.

42. Sieper J, Listing J, Poddubnyy D, Song IH, Hermann KG, Callhoff J, Syrbe U, Braun J and Rudwaleit M: Effect of continuous versus on-demand treatment of ankylosing spondylitis with diclofenac over 2 years on radiographic progression of the spine: Results from a randomised multicentre trial (ENRADAS). Ann Rheum Dis 75: 1438-1443, 2016.

(†) $\Theta$ This work is licensed under a Creative Commons Attribution-NonCommercial-NoDerivatives 4.0 International (CC BY-NC-ND 4.0) License. 